\title{
Increasing uptake of National Health Service Health Checks in primary care: a pragmatic randomized controlled trial of enhanced invitation letters in Northamptonshire, England
}

\section{A. Sallis ${ }^{1}$, N. Gold ${ }^{1,2}$, A. Agbebiyi ${ }^{1}$, R. J. E. James ${ }^{3}$, D. Berry ${ }^{4}$, A. Bonus ${ }^{4}$, I. Vlaev ${ }^{5}$, T. Chadborn ${ }^{1}$}

${ }^{1}$ Public Health England Behavioural Insights, Public Health England, London, UK

${ }^{2}$ Faculty of Philosophy, University of Oxford, Oxford, UK

${ }^{3}$ School of Psychology, University of Nottingham, Nottingham, UK

${ }^{4}$ Department of Health and Social Care, London, UK

${ }^{5}$ Behavioural Science Group, Warwick Business School, Coventry, UK

Address correspondence to Natalie Gold, E-mail: natalie.gold@phe.gov.uk

\begin{abstract}
Background Uptake of NHS Health Checks (NHSHCs) is sub-optimal. This study aimed to increase their uptake using behaviourally informed invitation letters.

Method Patients registered with 6 general practices in Northamptonshire, England who were eligible for an NHSHC between 10 February 2014 and 31 January 2015 were randomized monthly, using a random number generator, to three trial arms: control (standard invitation), sunk costs (resources already allocated) and counterargument (against common barriers to attendance). The outcome measure was uptake of NHSHC by 12 weeks after 31 January.

Results In total, 6331 patients were randomized. After exclusions, due to ineligibility for the NHSHC, data were analysed for $N=6313$ patients: $N=2123$ control; $N=2085$ counterargument; $N=2105$ sunk costs. Overall, 2364 (37.45\%) patients attended an NHSHC. Both intervention letters increased uptake compared to control, by $5.46 \%$ using counterargument (adjusted odds ratio (AOR) 1.32, CI 1.162-1.51, $p<0.001$ ) and $4.33 \%$ using sunk costs (AOR 1.246, CI 1.10-1.42, $p<0.001$ ), with no significant difference between the two.
\end{abstract}

Conclusion Behaviourally informed invitation letters, containing sunk costs or counterargument messages, can improve the uptake of NHSHCs The trial was registered with the International Standard Randomised Controlled Trial Registration Number Scheme (ISRCTN57110614).

Keywords Behavioural insights, Cardiovascular disease, Counterargument, Invitation letters, NHS Health Checks, Sunk costs, Uptake

\section{Introduction}

The NHS Health Check (NHSHC) programme is a cardiovascular disease (CVD) prevention programme, introduced in England in 2009. ${ }^{1,2}$ All GP-registered adults aged 40-74 who have not previously been diagnosed with CVD are invited to an NHSHC, which could be delivered in a primary care or other community setting, (for example a pharmacy or community provider) depending on the local delivery model, once every 5 years. ${ }^{2}$ The NHSHC assesses a person's risk of developing CVD using information on age, sex, family history, blood pressure, cholesterol, obesity, smoking, physical inactivity and alcohol intake. This information is then used to inform a patient-led discussion on what behavioural changes could be made to reduce a person's risk of CVD and, where appropriate, to agree medical interventions, e.g. statin prescribing.

Public Health England aspires to an uptake rate of $75 \%$ for NHSHCs. ${ }^{3}$ However, the national average uptake for 2014-2019 remains below that at 48\%, which includes checks that are done opportunistically, as well as checks that are done Sallis, A., Deputy Head of Behavioural Insights

Gold, N., Principal Behavioural Insights Advisor, Public Health England and Senior Research Fellow,

Agbebiyi, A., Public Health Speciality Registrar

James, R. J. E., Assistant Professor

Berry, D., Behavioural Insights Programme Manager, Department of Health

Bonus, A., Senior Behavioural Insights Researcher, Department of Health

Vlaev, I., Professor of Behavioural Science,

Chadborn, T., Head of Behavioural Insights and Evaluation Lead 
in response to an invitation. ${ }^{4,5}$ The response to an invitation letter is generally lower, according to published studies. Increasing uptake will help optimize the clinical and cost effectiveness of the programme. ${ }^{6}$ In 2013, PHE published an NHSHC 10-point implementation review and action plan, which included increasing uptake of health checks through behavioural insights interventions. ${ }^{7}$

There are a range of different invitation processes for NHSHCs, but the most common method is a letter from the patient's GP practice. Research is emerging which aims to optimize the content of these letters for improved uptake rates. $^{8-10}$ A randomized controlled trial (RCT) in Medway testing a behaviourally optimized version of the invitation letter increased NHSHC uptake by $4.2 \%$ compared to the national template letter in place at the time. ${ }^{8}$ The intervention letter was simplified (i.e. shorter) and included a clear behavioural instruction to book an NHSHC. The letter aimed to increase the personal salience of the appointment ('your appointment is due') and included a planning prompt in the form of a tear off slip to record the date, time and location of the appointment. A further RCT in Southwark, by the same authors, found a $4.9 \%$ increase in NHSHC uptake when using invitations that contained the same planning prompt as the previous trial but with added personalization (i.e. prepopulated with the patient's name, GP practice and practice address), and increases of up to $12 \%$ when patients were also sent SMS pre-notification and/or reminder messages.?

Theory and evidence suggest several other modifications to the content of the invitations that could have an effect. Providing counterarguments to debunk common myths has been successful at changing other health behaviours, for example increasing organ donation registrations. ${ }^{11}$ Qualitative and case studies show that the barriers to uptake of the NHSHC include a perception of no personal need ('I' $m$ fit and active, you should go when you're poorly'), ${ }^{12-14}$ the idea that it is irresponsible to take up NHS resources if one is not ill (' $I$ don't go to the doctor unless there is something wrong with me, you don't waste the doctor's time'), ${ }^{12,13}$ and fatalism about health outcomes ('if it's gonna happen, it's gonna happen') ${ }^{13}$ and the related view that learning their risks would not be helpful ('the knowledge will just make me anxious'). ${ }^{12,} 14$ Psychological models of the reflective processes underpinning health behaviour change predict that people are more likely to take action if an intervention increases either their perceived threat of getting a condition or their perceived efficacy, that they can take action to remove the threat ${ }^{15-18}$ (e.g. protection motivation theory and the health belief model). Providing a counterargument to the perception of no personal need should increase threat and providing a counterargument to fatalism about health outcomes should increase response efficacy, the belief that taking action will be effective in avoiding the threat of CVD.
Correcting perceptions about the threat of CVD and the efficacy of behaviour change should increase the perceived benefits of having an NHSHC, leading to greater attendance.

Another potential way to address beliefs about not wanting to waste doctors' time is to make salient the point that funding is already set aside for the NHSHC. Other research appealing to the public's desire not to waste NHS resources has been effective; specifying the cost of an NHS hospital appointment in a text message reminder to outpatients reduced the number of missed hospital appointments. ${ }^{19}$ This 'sunk cost effect' occurs when people's behaviour is affected by the fact that they have made a past investment of time or money, even though economic theory suggests that only costs that will be incurred in the future should affect decision-making. People exhibit the sunk cost fallacy because they do not want to be wasteful; ${ }^{20}$ the effect may also be related to 'loss aversion', whereby losses loom larger than gains, and the 'endowment effect', whereby the fact that they own something makes people value it more. ${ }^{21}$ Therefore, it seems likely that amending the invitation letter to imply that a patient has already been allocated an appointment and that the money has been put aside should increase uptake of the NHSHC.

This study tested the impact of behaviourally informed invitation letters on uptake of the NHSHC. One intervention used counterargument against common barriers to attendance and the other highlighted sunk-cost information to encourage people to make the most of scarce NHS resources.

\section{Method}

\section{Study design}

A pragmatic RCT design with two intervention arms and one control arm was employed with the patient as the unit of randomization.

\section{Participants and eligibility}

Patients from six General Practices in two NHS Trusts in Northamptonshire, England who were due for an NHSHC between 10 February 2014 and 31 January 2015 were eligible for the trial. Practices were recruited on the basis of their willingness to participate in the research and were invited by Northamptonshire Council and NHS Northamptonshire's R\&D collaborative. Practices with more than 9000 patients registered to them were prioritized for participation in the research.

\section{Procedure and randomization}

At the beginning of every month, a list of adults due for an NHSHC at each of the GP practices was generated, using specialized healthcare software. Patients on this list 


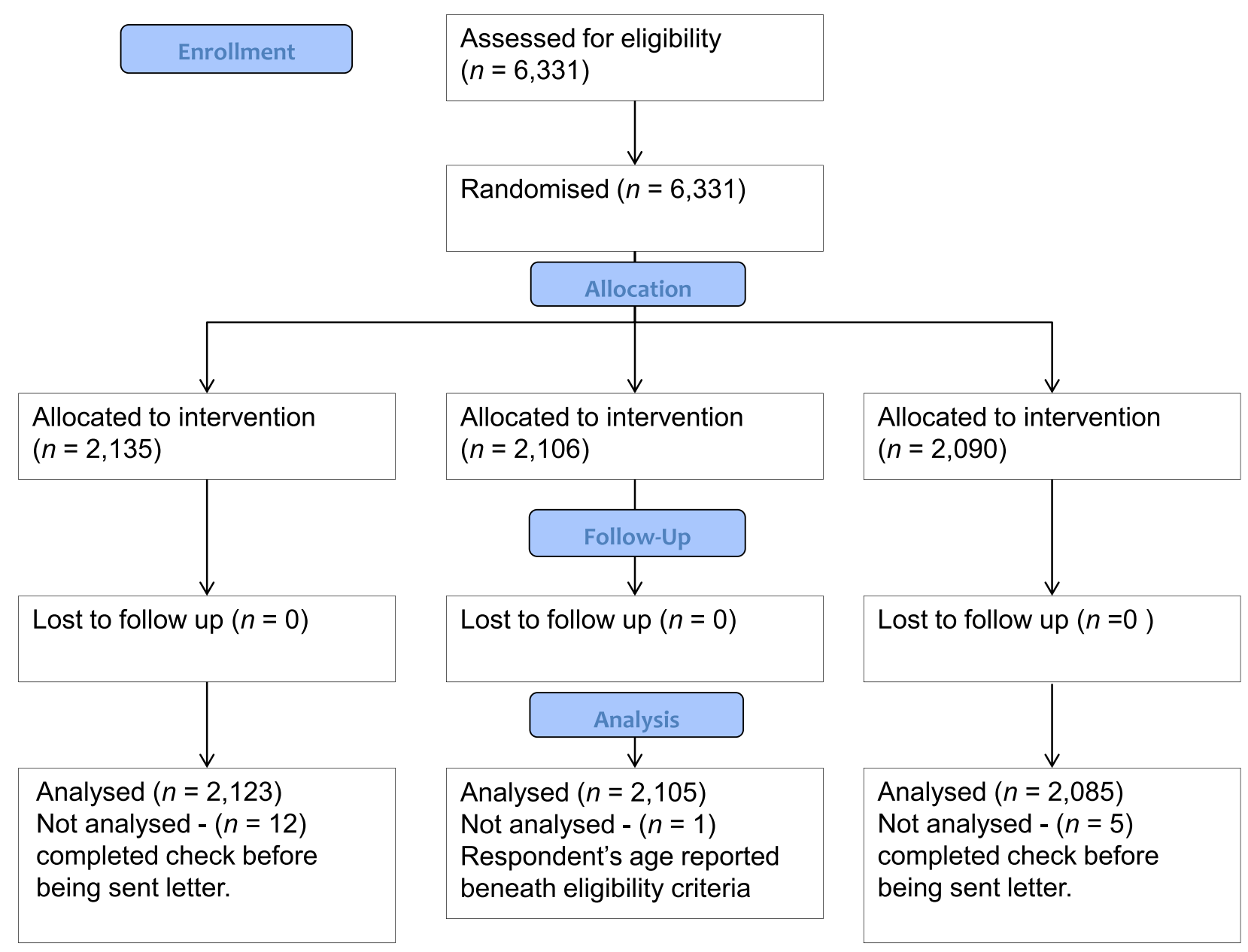

Fig. 1 Trial flowchart.

were randomly assigned to receive one of three different NHSHC invitation letters. Randomization was conducted monthly at each GP practice individually, by a researcher working with Northamptonshire's R\&D service, using a computerized random number generator (Microsoft Excel's random function). During the randomization process, the letters were referred to as 'Letter A', 'Letter B' and 'Letter C', and the researcher doing the randomization did not know to which conditions these referred or, therefore, to which condition participants were being assigned. Information on which letter each patient was sent was added to the patient's record. Eligible adults were sent invitation letters to attend an NHSHC. Patients who did not respond to the initial invitation letter were sent a standard reminder letter, eight weeks after the first letter. See Fig. 1 for a flowchart of the trial design.

Patients were blinded to the intervention; they did not know that other patients in the practice might have been sent a different invitation letter from them, nor did health check providers know what invitation any individual patient had been sent.

Researchers at the Department of Health and Social Care were sent the anonymized outcome data at the end of the trial, including patient demographic data (age, sex and ethnicity). Any patient who had received a letter and completed a check by the end of the data collection period was recorded as having completed an NHSHC. The trial was registered with the International Standard Randomised Controlled Trial Registration Number Scheme (ISRCTN57110614). Ethical approval was obtained from NHS Health Research Authority NRES committee (REF 13/SW/0293). We did not collect informed consent from participants because that would not have been practicable (we collected completely anonymized data from 6000 patients) and because that would have defeated the purpose of the trial (it is not possible to ask patients whether they want to receive a letter when investigating the effect of a letter on changing behaviour) and the NHS REC approved the trial on this basis. 
Table 1 Descriptive statistics of the gender, age, CVD risk and attendance rate of the three trial arms. Standard deviations are reported in brackets for continuous indicators

\begin{tabular}{llll} 
Measure & $\begin{array}{l}\text { Arm 1 (NHS template letter) } \\
(n=2123)\end{array}$ & Arm 2 (Sunk-costs letter) ( $n=2105)$ \\
\hline Attended NHS health check & 726 & 811 \\
Gender (female) & 804 & 806 & $2085)$ \\
Age & $57.21(7.98)$ & $56.86(7.97)$ & 796 \\
10-year CVD risk & $12.36(7.31)$ & $11.82(6.92)$ & $56.48(7.93)$
\end{tabular}

\section{Interventions}

Patients were randomly assigned to receive one of three letter invitations (see Supplementary Materials) inviting them to attend an NHSHC. The control letter was the national template letter at the time of the trial, which contains eight paragraphs explaining the purpose of the NHSHC, the eligible population, that taking early action can improve health, what happens at the appointment, how long it will take, that personalized advice will be received and that a leaflet is included providing further details. The first of the two intervention letters ('counterargument') included three boxed sections at the end of the letter detailing 'excuses' for not attending an NHSHC alongside a reply from the GP to counter this excuse. Each challenged one of three commonly reported reasons for non-attendance at health checks. ${ }^{12-14,22}$ The first argument explaining that the NHSHC can help to prevent the development of serious health conditions which take up lots of NHS resources was aimed at countering patients' beliefs about not wanting to waste NHS resources. The second argument explains that family history plays only a small role in the cause of heart attacks and that looking after your body is also important; this aimed to overcome the belief that family history dictates their future health outcomes. The final argument aimed to counter the belief that one should not go to a doctor unless they are ill by explaining that many health problems have hidden symptoms and that the NHSHC can help identify and address these. The second two statements attempt to increase perceived threat as well as increase response efficacy. The second intervention letter ('sunk-costs letter') was designed to encourage patients to make the most of scarce NHS resources by using the sunk-cost fallacy. The letter stated that 'Your GP has already set aside funding to pay for your appointment. Please take the time to attend.' The intervention letters also aimed to increase personal salience by suggesting that the appointment was due rather than simply being invited as well as being shorter and simpler and including behavioural instruction ('call to book your appointment') as per previous trials. $^{8,23}$ All three letters included the same leaflet that provided further information about the NHSHC.

\section{Outcome measure}

The outcome measure was attendance at the NHSHC recorded by individual practices. Data extraction was carried out 12 weeks after the end of the period when the letters were sent out. In addition, the GP practices provided anonymized age, sex and ethnicity data.

\section{Sample size}

A total of 6000 participants were required to obtain an estimated $80 \%$ power to detect a minimum of a $4 \%$ difference between groups at the 5\% significance level, at an estimated baseline level of attendance of $40 \%$.

\section{Statistical analyses}

A binomial logistic regression model was estimated in order to test whether the different letters increased uptake of the NHSHC. The outcome variable was whether the participant attended. The main independent variable was letter version; the national template was used as the reference category. A second adjusted mixed-effects model took account of demographics (age and sex). GP practice was also included in the model as a random effect. Because granular postcode data were not provided, Index of Multiple Deprivation (IMD) was estimated at the practice level. Therefore, there was perfect collinearity between GP practice and IMD, so only one of these variables could be entered into the regression analysis, and IMD was excluded. Ethnicity was not modelled in the analysis because levels of missing data systematically differed on the main outcome: $48.44 \%$ of individuals who did not take up the health check did not have any ethnicity data recorded about them, in contrast to the $97.57 \%$ of individuals who did attend a health check. In order to test whether the efficacy of the two interventions differed, a logistic regression model was estimated that excluded data from the control arm. 
Table 2 Table of respondents attending their health check by practice and trial arm

\begin{tabular}{llllll} 
& \multicolumn{5}{c}{ Number (percentage) of respondents attending HC } \\
\cline { 2 - 5 } Practice & Number of invitations sent & Control letter & Sunk-costs letter & Counterargument letter & Total \\
\hline 1 & 479 & $47(28.0 \%)$ & $56(32.0 \%)$ & $40(29.4 \%)$ & $143(29.9 \%)$ \\
2 & 835 & $134(47.7 \%)$ & $142(51.6 \%)$ & $135(48.4 \%)$ & $411(49.2 \%)$ \\
3 & 1277 & $161(37.2 \%)$ & $163(39.6 \%)$ & $186(43.1 \%)$ & $510(39.9 \%)$ \\
4 & 1136 & $130(34.5 \%)$ & $146(38.4 \%)$ & $164(43.3 \%)$ & $440(38.7 \%)$ \\
5 & 1377 & $115(25.0 \%)$ & $129(28.0 \%)$ & $132(28.9 \%)$ & $376(27.3 \%)$ \\
Total & 1209 & $139(34.4 \%)$ & $175(43.4 \%)$ & $170(42.3 \%)$ & $484(40.0 \%)$ \\
& 6313 & $726(34.2 \%)$ & $811(38.5 \%)$ & $827(39.7 \%)$ & $2364(37.5 \%)$
\end{tabular}

\section{Results}

A total of 6331 adults across the six practices were sent letters inviting them to book an appointment for the NHSHC. Five practices were based in NHS Nene CCG and one in NHS Corby CCG. Median practice list size was 12,288. Eighteen participants were excluded from the statistical analysis because they had attended an NHSHC prior to receiving a letter inviting them to book an appointment $(n=17)$ or because they were under the age of eligibility for the NHSHC $(n=1)$. This left 6313 participants: 2123 who received the standard template letter, 2085 who received the counterargument letter and 2105 who received the sunk-costs letter. There were 3907 males and 2406 females. More males were eligible for the NHSHC over the trial period. The mean age was 56.85 (S.D. $=7.97$ ). Chi-square tests showed no statistically significant differences between the trial arms on demographic factors. See Table 1 for a summary of the demographics of each arm.

Overall, $2364(37.45 \%)$ participants across the three arms attended an NHSHC. Of those who were sent the standard letter, $726(34.20 \%)$ participants attended, 827 (39.66\%) participants attended the NHSHC when sent the counterargument letter and $811(38.53 \%)$ attended when sent the sunk-costs letter. (See Table 2 for a breakdown by practice.) Compared to the control, this is a $5.46 \%$ absolute increase and a $16.0 \%$ relative increase with the counterargument letter and a $4.33 \%$ absolute increase and a $12.7 \%$ relative increase with the sunk-costs letter. A logistic regression shows that both intervention letters led to a significantly increased uptake of the NHSHC compared to the standard letter, with a $26.5 \%$ increase in the odds for a patient sent the counterargument letter (OR 1.27; 95\% CI 1.12-1.43; $p<0.001$ ) and a $21 \%$ increase in the odds of attendance for a patient sent the sunkcosts letter (OR 1.21; 95\% CI 1.064-1.37; $p=0.003$ ), see unadjusted model in Table 3 . The effects of the letters were
Table 3 Binary logistic regression model estimating the effectiveness of the intervention letters, with control letter as a baseline; adjusted model includes age (mean-centred at zero) and gender (baseline category male) as covariates and GP practice as a random effect

\begin{tabular}{llll} 
Indicator & Odds ratio & $95 \%$ C.I. & $p$ \\
\hline $\begin{array}{l}\text { Unadjusted model } \\
\text { Counterargument letter }\end{array}$ & 1.27 & $1.11,1.44$ & $<0.001$ \\
Sunk-costs letter & 1.21 & $1.07,1.38$ & 0.003 \\
Constant & 0.51 & $0.40,0.67$ & $<0.001$ \\
Adjusted model & & & \\
Counterargument letter & 1.32 & $1.16,1.51$ & $<0.001$ \\
Sunk-costs letter & 1.25 & $1.10,1.42$ & $<0.001$ \\
Female & 1.21 & $1.08,1.36$ & 0.001 \\
Age & 1.05 & $1.04,1.06$ & $<0.001$ \\
Constant & 0.46 & $0.33,0.63$ & $<0.001$ \\
& & &
\end{tabular}

similar in the model that controlled for demographics (see adjusted model in Table 3). In order to test whether the efficacy of the two interventions differed, a linear combination of variance (post-hoc test) on the adjusted model reported in Table 3, comparing the coefficients of the two intervention arms with the reference class as the sunk-costs letter, found no significant difference, OR1.06; 95\% CI 0.93-1.21; $z=0.91$; $p=.36$, indicating that there is no evidence in support of a difference between the intervention arms. In order to confirm this result, a logistic regression model was estimated that excluded data from the control arm. There was no evidence of a significant difference between the two intervention letters (adjusted odds ratio (AOR) 1.05; 95\% CI 0.93-1.19; $p=0.45$ ).

There were demographic differences in uptake (see the adjusted model in Table 3). Females were more likely to attend the NHSHC than males (AOR 1.21; $95 \%$ CI 
$1.08-1.36 ; p=0.001)$; in the sample as a whole, uptake amongst women was $44.56 \%$ compared to $33.07 \%$ amongst men. Uptake increased with age (AOR 1.05; 95\% CI $1.04-1.06 ; p<0.001)$. IMD was estimated at the practice level, so deprivation was perfectly correlated with practice. The intra-class correlation for the random effects is 0.033 , so $3.3 \%$ of the residual variance in the model is explained by practice level factors, including deprivation.

\section{Discussion}

\section{Main findings of this study}

Both intervention letters increased uptake of the NHSHC compared to the standard letter. Uptake rose by $5.46 \%$ with the counterargument letter and $4.33 \%$ with the sunk-costs letter. The difference in uptake between the two letters was not statistically significant. Uptake increased with age and females had $21 \%$ higher odds of attending than men. Uptake also depended on practice-level factors, which included relative deprivation.

\section{What is already known on this topic}

The absolute increases in attendance (5.46 and 4.33\% compared to the standard letter) are similar to the results of other studies that used behavioural science to enhance NHSHC invitation letters. Similarly, short letters using the same personal salience and behavioural instruction aspects but which were designed to address implementation intentions using planning prompts had increases of $4.2 \%$ in Medway and $4.9 \%$ in Southwark (the open letter without SMS prenotification or reminder). ${ }^{8}, 23$

The findings that women and older people are more likely to attend are consistent with national uptake statistics 24,25 and with the findings of previous studies that tested the effect of behaviourally enhanced invitation letters on the uptake of health checks. ${ }^{8}, 23$ The study found a practice level effect, which is consistent with other studies that have also found significant variance in uptake between practices. ${ }^{8,}$ 26-28 The practice-level effect in this study encompassed any effect of the level of deprivation, so it is not possible to make any statements about whether there was a higher uptake depending on the level of deprivation. However, there is evidence from other studies that patients in more affluent areas are more likely to attend health checks ${ }^{29}$ and specifically the NHSHC. ${ }^{30}$

\section{What this study adds}

This is the first study that we know of to correct misperceptions about the benefits of attending an NHSHC, in the counterarguments letter. We hypothesize the effect operates through reflective processes, as captured by the Health Belief Model and Protection Motivation Theory, increasing the perceptions of threat and response efficacy with respect to CVD. ${ }^{15-18}$ Three counterarguments were used, targeting three different barriers to NHS HC attendance, which were designed to appeal to a broad audience. There is the potential for future work to test messages that are tailored for different target audiences.

The two previous studies amending NHSHC letters and successfully increasing uptake of the NHSHC used planning prompts aimed at overcoming the intention-behaviour gap.

23 The present study lends further support to strategies aimed at altering aspects of reflective motivational states. However, another RCT targeting reflective beliefs using behavioural techniques found no effect on uptake of NHSHC. ${ }^{10}$ Eligible patients were sent a questionnaire regarding their attitudes, intentions subjective norms, perceived behavioural control and anticipated regret regarding attendance at an NHSHC one week before the invitation letter, to trigger the questionbehaviour effect, whereby asking questions about a behaviour is hypothesized to increase the likelihood that it will be performed. The authors propose several pathways by which this is thought to be effective: by (i) increasing the accessibility of certain beliefs related to the NHSHC, thereby making it more likely the behaviour will be performed, (ii) creating cognitive dissonance (a discrepancy between intended and actual behaviour) and/or (iii) enabling the patient to create mental representations or behavioural scripts which can be recalled and reactivated when the invitation letter is received. It is also possible that this intervention failed to increase uptake because it drew attention to potentially negative beliefs about attendance at the NHSHC and did not subsequently bolster response or self-efficacy following this. The questionnaire was sent a week before the invitation letter, so the intervention was not immediately connected to the opportunity for taking action and booking an NHSHC like the present study and other two successful studies in this area. ${ }^{8,23}$

The present study also found that emphasizing sunk costs was effective at increasing uptake, by saying that money had been put aside for the patient's health check and implying that the patient would waste that money if $\mathrm{s} /$ he did not attend. A previous study found that specifying the estimated cost of a hospital outpatient appointment $(£, 160)$ in a reminder text decreased the rate of people who did not attend from $11.1 \%$ (with the standard appointment reminder text) to $8.4 \% .{ }^{19}$ The same study also found that a general costs message, stating that 'Not attending costs the NHS money' was less effective than quoting specific costs. Here it was not possible to put a precise cost on an NHSHC and the cost would have been far less than the cost of an outpatient appointment, therefore 
poetically diluting its effects, and therefore a general costs message was used. The general costs message in the present study was additionally reinforced by the implication that the costs were sunk, and the results showed that a general sunkcost message can be as effective as counterarguments.

The counterarguments letter mainly emphasized the personal health benefits of attending the NHSHC, whilst the sunk-costs letter emphasized the costs to the NHS of not attending. Both were effective. This contrasts with a study on medicine adherence, which found that emphasizing personal health costs was effective ('Not taking my medication as prescribed could risk my health. I want to do all I can to improve my health, so I commit to taking this medication exactly as prescribed'), but emphasizing social costs was not ('The NHS loses $f_{3}, 30$ million per year from wasted medication. I want to do my bit to support the NHS, so I commit to taking this medication exactly as prescribed'). ${ }^{31}$ However, the medicine adherence study did not utilize the behavioural lever of sunk costs. Further, the medicine adherence study stated the total annual cost to the NHS of wasted medication, so the individual patient's contribution to this total may have seemed miniscule in comparison, giving a perception that behaviour change by one individual would make little difference. In contrast, the sunk-costs letter emphasized the cost to the NHS of the specific individual patient who received the invitation not attending their NHSHC, thereby increasing their sense of personal responsibility for any wasted money.

\section{Limitations of this study}

In this study, data on ethnicity were often missing and ethnicity was not included in the data analysis because non-attendees were less likely to have had ethnicity data recorded than attendees. Having ethnicity recorded has previously been found to be associated with higher uptake compared to patients whose ethnicity was not recorded. ${ }^{32,} 33$ Ethnicity data need to be collected accurately if strategies for increasing uptake are to be targeted at high risk groups. Ethnicity is an important factor in vascular and renal risk, as South Asians have a higher risk of diabetes ${ }^{34,35}$ and CVD. ${ }^{36}$

\section{Conclusions}

This study showed that behaviourally optimized invitation letters can improve attendance at the NHSHC. Attendance increased when patients were given counterarguments to common barriers to attendance, correcting their misperceptions and increasing the perceived benefits of attending. Attendance also increased when the invitation letter implied sunk-costs, that money had been put aside, so that non- attendance would cost the NHS money. Further work could test the combination of different messages and their interaction with the planning prompts that have been found to be effective in previous trials. It could also be interesting to explore the tailoring the message content to different groups.

\section{Supplementary data}

Supplementary data are available at the Journal of Public Health online.

\section{Acknowledgements}

The authors are grateful to J.W., PHE National Lead for CVD Prevention, for commissioning this work and to K.T., PHE Deputy National Lead for NHS HCs, for comments on the manuscript. We are grateful to K.V. and S.P.-H. for their help in implementing the trial. I.V. is supported by the National Institute for Health Research Collaboration for Leadership in Applied Health Research and Care West Midlands (CLAHRCWM) initiative.

\section{Author contributions}

AS, DB \& $\mathrm{AB}$ conceived and designed the study methodology and interventions. $\mathrm{AB} \& \mathrm{DB}$ executed the study. RJ analysed the data and wrote an initial draft of sections of the manuscript. AA produced an initial draft of key sections of the manuscript with supervision and guidance from AS. AS drafted sections of the manuscript and took overall editorial responsibility for the paper. NG re-drafted and critically revised the manuscript. IV advised on the write-up and the interpretation of results. TC provided advice, guidance and resources for analysis and paper drafting. All authors contributed to interpretation of results and agreed to the final version.

\section{References}

1 Department of Health. Putting Prevention First-NHS Health Check: Vascular Risk. Assessment and Management, Best Practice Guidance. London: Department of Health, 2009.

2 Public Health England. NHS Health Check, Best Practice Guidance. London: HMSO, 2016.

3 Waterall J, Greaves F, Kearney M, Fenton KA. Invited debate: NHS health check: an innovative component of local adult health improvement and well-being programmes in England. J Public Health 2015;37:177-84.

4 Fingertips [https://fingertips.phe.org.uk/profile/nhs-health-checkdetailed/data\#page/0]. 
5 NHS Health Check [http://www.healthcheck.nhs.uk/commissione rs_and_providers/data/].

6 Public Health England. NHS Health Check Programme Standards. London: HMSO, 2014

7 Public Health England. NHS Health Check Implementation Review and Action Plan. London: Public Health England, 2013.

8 Sallis A, Bunten A, Bonus A et al. The effectiveness of an enhanced invitation letter on uptake of National Health Service Health Checks in primary care: a pragmatic quasi-randomised controlled trial. $B M C$ Fam Pract 2016;17:35

9 Sallis A, Sherlock J, Bonus A et al. Pre-notification and reminder SMS text messages with behaviourally informed invitation letters to improve uptake of NHS health checks: a factorial randomised controlled trial. BMC Public Health 2019;19:1162.

10 McDermott L, Wright AJ, Cornelius V et al. Enhanced invitation methods and uptake of health checks in primary care. Rapid randomised controlled trial using electronic health records. Health Technol Assess 2016;20:385.

11 Siegel JT, Alvaro EM, Crano WD et al. A quasi-experimental investigation of message appeal variations on organ donor registration rates. Health Psychol 2008;27:170.

12 Burgess C, Wright AJ, Forster AS et al. Influences on individuals' decisions to take up the offer of a health check: a qualitative study. Health Expect 2015;18:2437-48.

13 Jenkinson CE, Asprey A, Clark CE, Richards SH. Patients' willingness to attend the NHS cardiovascular health checks in primary care: a qualitative interview study. BMC Fam Pract 2015;16:33.

14 Ellis N, Gidlow C, Cowap L et al. A qualitative investigation of nonresponse in NHS health checks. Arch Public Health 2015;73:14.

15 Rogers RW. A protection motivation theory of fear appeals and attitude change. J Psychol 1975;91:93-114.

16 Hochbaum GM. Public Participation in Medical Screening Programs: A SocioPsychological Study. Washington, DC: US Department of Health. Public Health Service ...: Education, and Welfare, 1958.

17 Janz NK, Becker MH. The health belief model: a decade later. Health Educ Q 1984;11:1-47.

18 Rosenstock IM. Historical origins of the health belief model. Health Educ Monogr 1974;2:328-35.

19 Hallsworth M, Berry D, Sanders M et al. Stating appointment costs in SMS reminders reduces missed hospital appointments: findings from two randomised controlled trials. PLoS One 2015;10:e0137306.

20 Arkes HR, Blumer C. The psychology of sunk cost. Organ Behav Hum Decis Process 1985;35:124-40.
21 Thaler R. Toward a positive theory of consumer choice. J Econ Behav Organ 1980;1:39-60.

22 NHS Greenwich. Understanding Target Groups Perceptions of the NHS Greenwich Health Check PLUS Programme, 2011.

23 Labeit A, Peinemann F, Baker R. Utilisation of preventative health check-ups in the UK: Findings from individual-level repeated cross-sectional data from 1992 to 2008. BMJ Open 2013;3: $\mathrm{e} 003387$.

24 Robson J, Dostal I, Sheikh A et al. The NHS health check in England: an evaluation of the first 4 years. BMJ Open 2016;6:e008840.

25 Attwood S, Morton K, Sutton S. Exploring equity in uptake of the NHS health check and a nested physical activity intervention trial. $J$ Public Health (Oxf) 2016;38:560-8.

26 Artac M, Dalton AR, Majeed A et al. Uptake of the NHS health check programme in an urban setting. Fam Pract 2013;30:426-35.

27 Cochrane T, Gidlow CJ, Kumar J et al. Cross-sectional review of the response and treatment uptake from the NHS health checks programme in Stoke on Trent. J Public Health (Oxf) 2013;35:92-8.

28 Waller D, Agass M, Mant D et al. Health checks in general practice: another example of inverse care? BMJ 1990;300:1115-8.

29 Cochrane T, Davey R, Iqbal Z et al. NHS health checks through general practice: randomised trial of population cardiovascular risk reduction. BMC Public Health 2012;12:944.

30 Jachimowicz JM, Gladstone JJ, Berry D et al. Making medications stick: improving medication adherence by highlighting the personal health costs of non-compliance. Behav Public Policy 1-21. doi:10.1017/bpp.2019.1

31 Artac M, Dalton AR, Babu $\mathrm{H}$ et al. Primary care and population factors associated with NHS health check coverage: a national cross-sectional study. J Public Health (Oxf) 2013;35:431-9.

32 Lambert A, Burden A, Chambers J, Marshall T. Cardiovascular screening for men at high risk in heart of Birmingham teaching primary care trust: The 'deadly Trio'programme. J Public Health 2011;34:73-82.

33 Smith S, Waterall J, Burden AF. An evaluation of the performance of the NHS health check programme in identifying people at high risk of developing type 2 diabetes. BMJ Open 2013;3:e002219.

34 Smith S, Waterall J, Burden A. Correction. An evaluation of the performance of the NHS health check programme in identifying people at high risk of developing type 2 diabetes. BMJ Open 2015;5:e002219corr002211-1.

35 Dalton AR, Bottle A, Soljak M et al. Ethnic group differences in cardiovascular risk assessment scores: national cross-sectional study. Ethn Health 2014;19:367-84. 